\title{
A four-gene signature for prognosis in breast cancer patients with hypermethylated IL15RA
}

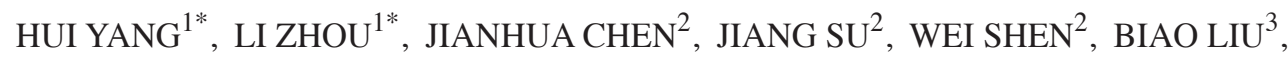 \\ JUNDONG ZHOU $^{4}$, SHIYOU YU ${ }^{2}$ and JUN QIAN ${ }^{1}$ \\ Departments of ${ }^{1}$ Medical Oncology, ${ }^{2}$ Surgical Oncology, ${ }^{3}$ Pathology and ${ }^{4}$ Radiotherapy, \\ Nanjing Medical University Suzhou Hospital, Suzhou Cancer Center, Suzhou, Jiangsu 215001, P.R. China
}

Received May 3, 2018; Accepted December 13, 2018

DOI: $10.3892 / 01.2019 .10137$

\begin{abstract}
Previous studies have revealed that upregulation of interleukin 15 receptor $\alpha$ (IL15RA) contributes to improved prognosis of breast cancer. The present study aimed to elucidate the molecular mechanisms underlying the antitumor effect induced by IL15RA upregulation, and to identify a gene signature capable of predicting the survival of patients with breast cancer. Using paired gene expression and methylation data of breast cancer samples from The Cancer Genome Atlas data portal, differentially expressed genes (DEGs) were identified in hypermethylated and hypomethylated IL15RA breast cancer samples. Furthermore, a gene signature-based risk-scoring model was developed according to the Cox regression coefficients of survival-associated DEGS. The gene signature was applied to classify patients with breast cancer and hypermethylated IL15RA into two risk groups via Kaplan-Meier survival analysis of overall survival (OS) time. Functional enrichment analysis was conducted to decipher the biological roles of the DEGs between the two risk groups. A total of 326 DEGs were present in the hypomethylation and hypermethylation samples compared with in the normal samples. A four-gene signature [SH3 and cysteine rich domain 2 (STAC2), proline rich 11 (PRR11), homeobox C11 (HOXC11) and nucleolar and spindle associated protein 1 (NUSAP1)] was identified as able to successfully separate patients with
\end{abstract}

Correspondence to: Dr Shiyou Yu, Department of Surgical Oncology, Nanjing Medical University Suzhou Hospital, Suzhou Cancer Center, 16 Baita West Road, Suzhou, Jiangsu 215001, P.R. China

E-mail: njshiyouyu@sina.com

Dr Jun Qian, Department of Medical Oncology, Nanjing Medical University Suzhou Hospital, Suzhou Cancer Center, 16 Baita West Road, Suzhou, Jiangsu 215001, P.R. China

E-mail: byqj@medmail.com.cn

${ }^{*}$ Contributed equally

Key words: hypermethylation, survival, risk score, Gene Ontology, pathway breast cancer and hypermethylated IL15RA into two risk groups with significantly different OS time. The signature revealed similar predictive performance in an independent set. Significant enrichment of the 'receptor interaction' and 'cell adhesion molecules (CAM)' pathways, which involved the DEGs, occurred between the two risk groups. These findings suggested that IL15RA may participate in the regulation of STAC2, PRR11, HOXC11, NUSAP1, and 'ECM-receptor interaction' and 'cell adhesion molecules' pathways, and therefore in the suppression of breast cancer development and progression. The four-gene signature may have potential prognostic value for breast cancer.

\section{Introduction}

Breast cancer is the most common type of cancer in women, accounting for $25 \%$ of all cases of cancer (1). According to gene expression profiles, the molecular features of breast cancer are divided as follows: Human epidermal growth factor receptor 2-positive, a basal subtype and two luminal subtypes (2). In addition, infiltration of various types of immune cells is commonly observed in breast cancer. For instance, emerging evidence has demonstrated that lymphocytic infiltration in breast cancer is generally associated with a favorable prognosis (3-6).

Interleukin 15 receptor $\alpha$ (IL15RA) is a component of the IL15R, which shares a $\beta$ and $\gamma$ subunit with the IL2 receptor (7). IL15-dependent signaling regulates the activation and proliferation of T cells and natural killer cells, and modulates the adaptive immune response (8). Marra et al (9) demonstrated that IL15 and IL15RA are frequently upregulated in breast cancer and breast cancer cell lines. The authors suggested that upregulation of IL15 and IL15RA induces an antitumor immune response by activating peripheral blood mononuclear cell synthesis, and subsequently improves the prognosis of patients with lymphocyte-enriched breast cancer. However, the underlying mechanisms of the effects of IL15RA expression on breast cancer remain unknown.

The present study aimed to investigate the molecular mechanisms underlying the antitumor effects induced by IL15RA upregulation and to identify a gene signature for breast cancer prognosis. Since IL15RA/cg09290866 hypermethylation leads to the suppression of IL15RA expression, and hypomethylation 
elevates the expression of IL15RA, the present study aimed to highlight the differentially expressed genes (DEGs) detected in hypermethylated and hypomethylated IL15RA breast cancer samples compared to normal samples. Univariate Cox regression analysis identified the prognosis-associated DEGs. In silico analyses of the expression profiles of these prognosis-associated DEGs enabled the generation of a four-gene signature, capable of predicting the odds of patient outcome, in the training and test sets. The four-gene signature may allow the classification of patients with IL15RA hypermethylation in the training set into a high-risk group and a low-risk group with significantly different survival times. In addition, the DEGs between the two risk groups were identified, and their possible biological roles were studied using Gene Ontology (GO) function and Kyoto Encyclopedia of Genes and Genomes (KEGG) pathway enrichment analyses.

\section{Materials and methods}

The Cancer Genome Atlas (TCGA) and Gene Expression Omnibus (GEO) datasets. The present study acquired the gene expression and methylation data of 316 breast cancer samples and 21 normal tissue samples from The Cancer Genome Atlas (TCGA) data portal (https://portal.gdc.cancer.gov/projects) based on Illumina Methylation 450 platform and Illumina HiSeq 2000 RNA Sequencing (Illumina, Inc., San Diego, CA, USA). These samples were defined as the training set (TCGA set). The corresponding clinical characteristics are shown in Table I.

The GSE37751 dataset (10), including the gene expression data of human breast tumor samples, and the GSE39004 dataset, (10) consisting of DNA methylation profiles of the same human breast tumor samples, were downloaded from the National Center of Biotechnology Information (NCBI) GEO database (http://www.ncbi.nlm.nih.gov/geo/). A total of 57 samples with paired gene expression and DNA methylation data were selected as the test set (GEO set). Clinical characteristics of the test set are displayed in Table I.

DEGs screening. According to the methylation value of IL15RA/cg09290866 ( $\beta$-value), samples from the training set were categorized into a hypermethylation group $(\beta$-value $\geq 0.3)$ and a hypomethylation group ( $\beta$-value $<0.3)$. A false discovery rate $(\mathrm{FDR})<0.05$ and $\mid \log$ fold change $(\mathrm{FC}) \mid>0.585$ were considered as the strict cutoff thresholds, and the DEGs were subsequently screened by comparing the DEGs in the hypermethylation or hypomethylation samples with those in the normal samples using Limma package (11) (http://www. bioconductor.org/packages/release/bioc/html/limma.html) in R3.1.0 language. The overlapping DEGs in both hypermethylation and hypomethylation samples were selected for further analysis.

Association of DEGs with patient survival. Univariate Cox regression analysis (12) was performed to evaluate the association of the overlapping DEGs with patient survival using survival package (https://cran.r-project.org/web/packages/survival/index.html) in R3.1.0 language. The DEGs that were significantly associated with patient survival were identified $(\log$-rank $\mathrm{P}<0.05)$ and ordered.
Risk score. The risk score was calculated for each patient based on the linear combination of expression levels of the DEGs with the regression coefficient as follows:

\section{Risk score $=\beta$ gene $1 \mathrm{x}$ exprgene $1+\beta$ gene $2 \mathrm{x}$ exprgene $2+\cdots+\beta$ gene $n \mathrm{x}$ exprgene $\mathrm{n}$}

where ' $\beta$ gene n' describes the estimated regression coefficient of the gene $n$ derived from the univariate Cox regression analysis, and 'exprgene n' describes the expression level of the gene $\mathrm{n}$.

By considering the median risk score of the hypermethylation group as the cutoff value, the hypermethylation samples in the training set were divided into a high-risk and a low-risk subgroup. By applying the $\beta$-value derived from the training set, the samples in the test set were also classified by risk score into a high-risk and a low-risk subgroup.

Statistical analysis. The overall survival (OS) time of the risk groups was analyzed using Kaplan-Meier (KM) survival analysis followed by log-rank test. Multivariate Cox regression analysis and data stratification analysis were conducted to determine the association of risk score with other clinical characteristics in TCGA and GEO sets. $\mathrm{P}<0.05$ was considered to indicate a statistically significant difference. Hazard ratios (HR) and 95\% confidence intervals (CI) were calculated.

Functional enrichment analysis. In the training set, the DEGs between the high-risk and the low-risk groups were also identified using the Limma package, with the threshold set at FDR $<0.05$. Correlations of the DEGs selected with risk score were characterized using cor function (https://www.rdocumentation.org/packages/stats/versions/3.4.1/topics/cor) in $\mathrm{R}$ language. The DEGs with correlation coefficient $0-1$ were defined as positive DEGs, whereas the DEGs with correlation coefficient -1-0 were defined as negative DEGs. The positive or negative DEGs were ranked according to their $r$. The top 20 positive DEGs and the top 20 negative DEGs underwent GO (13) function and KEGG (14) pathway enrichment analyses using the Database for Annotation, Visualization and Integrated Discovery (DAVID) (15). There are three types of GO terms, including biological processes (BP), cellular compartment (CC), and molecular function (MF). GO terms and KEGG pathways with FDR $<0.05$ were considered as significant functional annotations.

\section{Results}

Identification of DEGs. There were 226 hypermethylation samples $(\beta$-value $\geq 0.3), 90$ hypomethylation samples $(\beta$-value $<0.3)$ and 21 normal samples in the training set. $A$ total of 326 overlapping DEGs $(\mathrm{FDR}<0.05, \mid \log \mathrm{FCl}>0.585)$ were identified in the hypomethylation and hypermethylation samples relative to the normal samples.

Development of a four-gene signature-based risk-scoring model. The genes associated with prognosis were selected from the overlapping DEGs previously identified in the training set using univariate Cox regression analysis. These genes were then used to build a risk-scoring model for prognosis. 
Table I. Clinical summary of patients in TCGA and GEO datasets.

\begin{tabular}{lcc}
\hline Clinical features & TCGA $(\mathrm{n}=316)$ & GSE39004 (n=57) \\
\hline Pathologic_M (M0/M1/MX) & $299 / 9 / 8$ & - \\
Pathologic_N (N0/N1/N2/N3/NX) & $167 / 95 / 25 / 21 / 8$ & - \\
Pathologic_T (M1/M2/M3/M4/MX) & $84 / 187 / 28 / 16$ & $3 / 40 / 14 / 0 / 0$ \\
Pathologic_stage (I/II/III/IV/-) & $57 / 185 / 52 / 9 / 13$ & $17 / 38 / 2$ \\
Age (>60/_60/-, years) & $154 / 162$ & $29 / 28$ \\
ER_Status (positive/negative/-) & $238 / 69 / 9$ & - \\
HER2_Status (positive/negative/-) & $56 / 243 / 17$ & - \\
PR_Status (positive/negative/-) & $201 / 106 / 9$ & - \\
Radiation_therapy (Yes/No/-) & $6 / 18 / 292$ & - \\
Pharmaceutical_therapy (Yes/No) & $11 / 14 / 291$ & - \\
Hormone_therapy (Yes/No) & $36 / 280$ & $23 / 33 / 1$ \\
Mortality (Dead/Alive) & $29.07 \pm 34.73$ & $57.61 \pm 38.03$ \\
Overall survival days (months, mean \pm SD) & \\
\hline
\end{tabular}

'-', data unavailable. ER, estrogen receptor; HER2, human epidermal growth factor receptor; GEO, Gene Expression Omnibus; PR, progesterone receptor; SD, standard deviation; TCGA, The Cancer Genome Atlas.
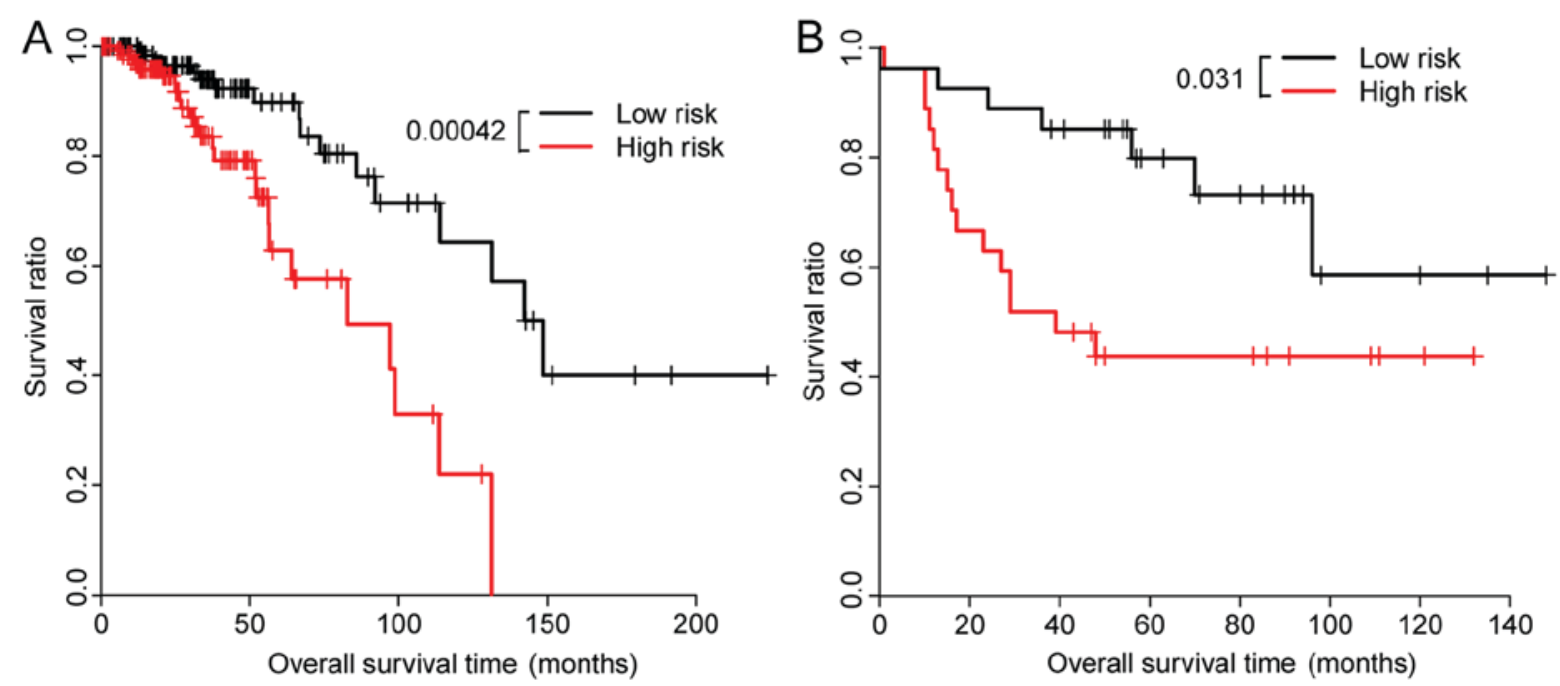

Figure 1. Kaplan-Meier plots of different risk groups in (A) The Cancer Genome Atlas set and (B) Gene Expression Omnibus set stratified by risk score.

Eventually, the SRC homology 3 (SH3) domain, SH3 and cysteine rich domain 2 (STAC2), proline rich 11 (PRR11), homeobox $\mathrm{C} 11$ (HOXC11) and nucleolar and spindle associated protein 1 (NUSAP1) genes were selected to calculate the risk score based on their Cox regression coefficients as follows:

$$
\begin{gathered}
\text { Risk score }=-1.744862 \times \text { Exp STAC } 2+-0.969505 \times \text { Exp } \\
K C N D 3+1.404081 \times E x p \text { HOXC11 }+-4.4925 \times \operatorname{Exp} \text { NUSAP1 }
\end{gathered}
$$

A risk score was assigned to each patient in the hypermethylation group. By using the 50th percentile cutoff of risk score as the threshold, all hypermethylation samples were classified into a high-risk and a low-risk subgroup. The low-risk subgroup exhibited significantly elongated survival time compared with the high-risk subgroup (median OS: 647 days vs. 92 days; $\mathrm{P}=0.00042$; Fig. 1A).
To validate the prognostic performance of the four-gene signature in the test set, the patients with breast cancer in the test set were also dichotomized into low-risk and high-risk subgroups using the 50th percentile cutoff of risk score as the threshold. Similarly, better survival was observed in the low-risk group compared with the high-risk group (median OS: 1,792 days vs. 1,209 days; $\mathrm{P}=0.031$; Fig. $1 \mathrm{~B}$ ).

Expression of the four prognostic genes in different risk groups. As shown in Fig. 2, the high-risk group presented significantly increased expression levels of PRR11, HOXC11 and NUSAP1, and significantly decreased expression of STAC2 compared to the low-risk subgroup in the training set (Fig. 2A; PRR11, $\mathrm{P}<0.005$; STAC2, $\mathrm{P}<0.005$; HOXC11, $\mathrm{P}<0.005$; NUSAP1, $\mathrm{P}<0.005$ ) and the test set (Fig. 2B; PRR11, P<0.005; STAC2, $\mathrm{P}<0.005$; HOXC11, $\mathrm{P}<0.05$; NUSAP1, $\mathrm{P}<0.001)$. 

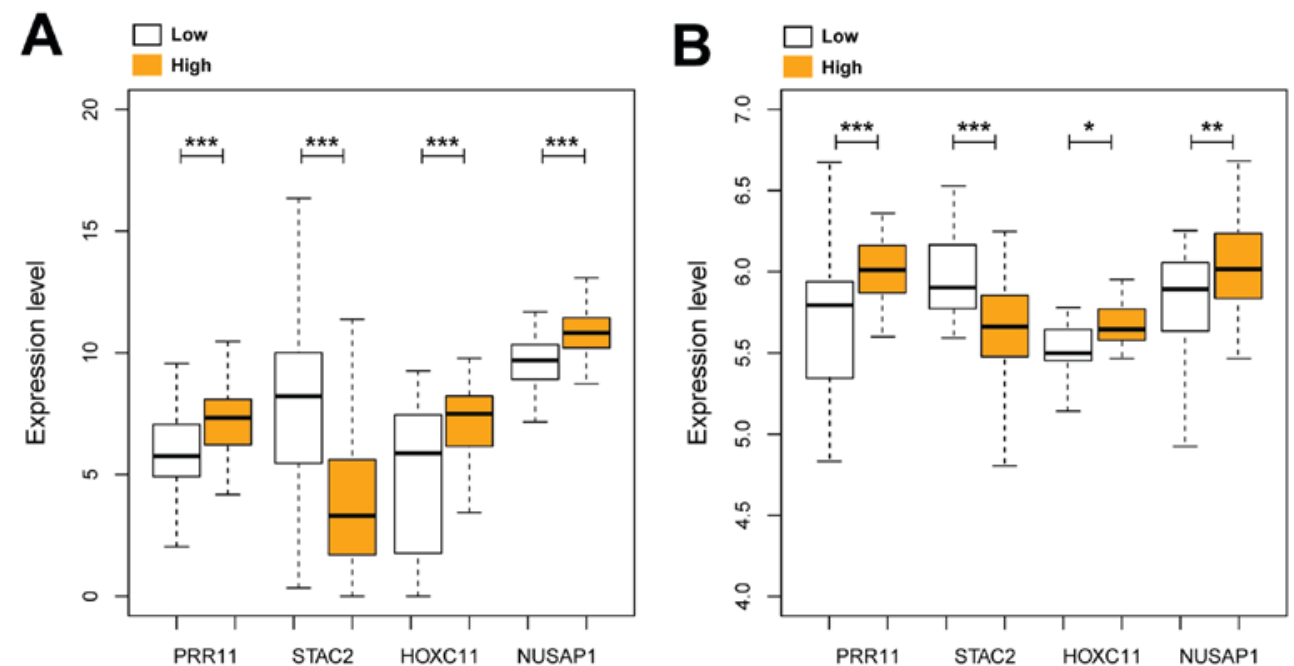

Figure 2. Expression of the four signature genes in different risk groups of (A) The Cancer Genome Atlas set and (B) Gene Expression Omnibus set. "P $<0.05$, ${ }^{* *} \mathrm{P}<0.01,{ }^{* * *} \mathrm{P}<0.005$. HOXC1, homeobox C11; NUSAP1, nucleolar and spindle associated protein 1; PRR11, proline rich 11; STAC2, SH3 and cysteine rich domain 2 .

A
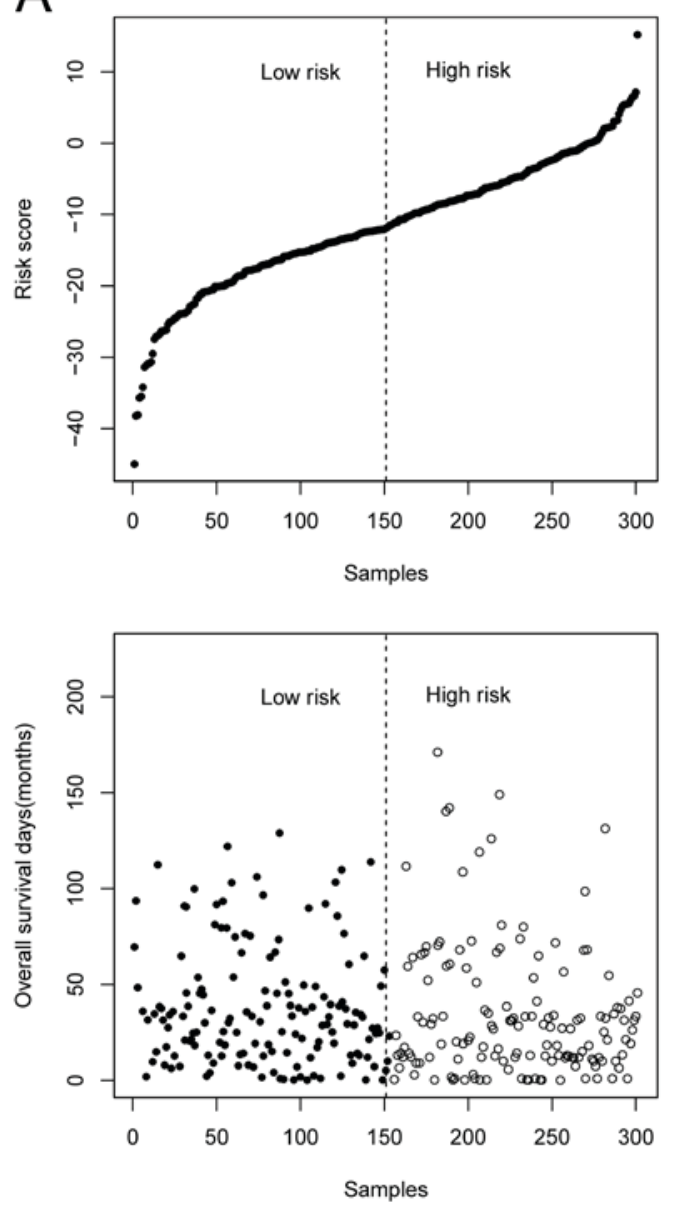

High expression

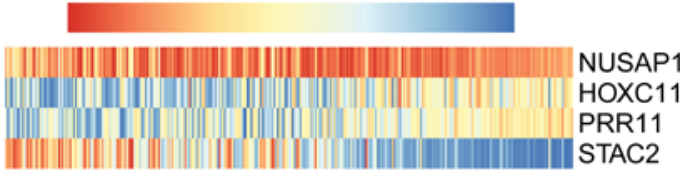

$\mathrm{B}$
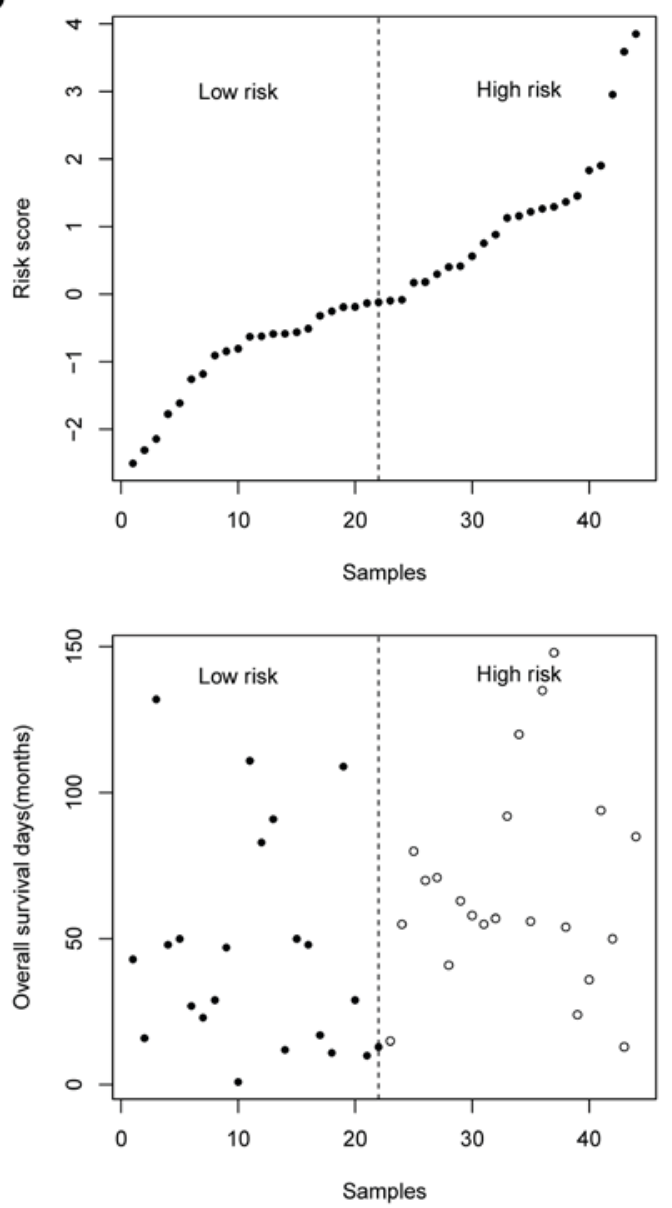

High expression

Low expression

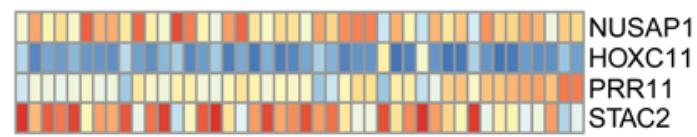

Figure 3. Four-gene risk score distribution, overall survival of patients and a heatmap displaying the expression of the four genes in (A) The Cancer Genome Atlas set and (B) Gene Expression Omnibus set. HOXC1, homeobox C11; NUSAP1, nucleolar and spindle associated protein 1; PRR11, proline rich 11; STAC2, SH3 and cysteine rich domain 2. 
Table II. Regression coefficients from univariate and multivariate Cox regression analysis.

\section{A, TCGA}

\begin{tabular}{|c|c|c|c|c|}
\hline \multirow[b]{2}{*}{ Variable } & \multicolumn{2}{|c|}{ Univariate Cox } & \multicolumn{2}{|c|}{ Multivariable Cox } \\
\hline & $\mathrm{P}$-value & HR (CI 95\%) & P-value & HR (CI 95\%) \\
\hline pathologic_T & 0.156 & $1.398(0.881-2.220)$ & - & - \\
\hline ER_Status & 0.549 & $0.771(0.328-1.808)$ & - & - \\
\hline HER2_Status & 0.731 & $0.831(0.288-2.396)$ & - & - \\
\hline pathologic_M & 0.014 & $3.480(1.284-9.430)$ & 0.094 & $2.941(1.676-2.947)$ \\
\hline pathologic_N & 0.017 & $1.517(1.077-2.136)$ & 0.072 & $1.009(0.656-1.846)$ \\
\hline Age & 0.048 & $1.030(1.000-1.060)$ & 0.126 & $1.026(0.993-1.061)$ \\
\hline PR_Status & 0.025 & $0.426(0.202-0.897)$ & 0.053 & $0.304(0.132-0.698)$ \\
\hline pathologic_stage & $<0.005$ & $1.813(1.200-2.738)$ & $<0.005$ & $1.198(0.487-2.947)$ \\
\hline Risk score & $<0.005$ & $1.086(1.044-1.128)$ & $<0.005$ & $1.069(1.023-1.117)$ \\
\hline
\end{tabular}

\section{B, GSE39004}

\begin{tabular}{|c|c|c|c|c|}
\hline \multirow[b]{2}{*}{ Variable } & \multicolumn{2}{|c|}{ Univariate Cox } & \multicolumn{2}{|c|}{ Multivariable Cox } \\
\hline & P-value & $\mathrm{HR}(\mathrm{CI})$ & P-value & $\mathrm{HR}(\mathrm{CI})$ \\
\hline Ethnicity & 0.185 & $0.515(0.193-1.376)$ & - & - \\
\hline ER_Status & 0.171 & $0.521(0.205-1.326)$ & - & - \\
\hline Triple & 0.818 & $1.128(0.402-3.167)$ & - & - \\
\hline Grade & 0.302 & $1.424(0.728-2.787)$ & - & - \\
\hline Neoadjuvant-therapy & 0.621 & $0.601(0.0797-1.527)$ & - & - \\
\hline Hormone-therapy & 0.262 & $0.581(0.225-1.501)$ & - & - \\
\hline Chemotherapy & 0.204 & $0.547(0.216-1.387)$ & - & - \\
\hline Stage & 0.045 & $2.646(1.023-3.845)$ & 0.0663 & $2.458(0.941-3.42)$ \\
\hline Age & 0.016 & $1.039(1.007-1.071)$ & 0.0125 & $1.036(1.008-1.128)$ \\
\hline Risk score & 0.029 & $0.827(0.582-1.175)$ & 0.0205 & $0.780(0.534-1.141)$ \\
\hline
\end{tabular}

Bold indicates significant differences. CI, confidence interval; ER, estrogen receptor; HER2, human epidermal growth factor receptor; HR, hazard ratio; PR, progesterone receptor; SD, standard deviation; TCGA, The Cancer Genome Atlas.

Fig. 3 displayed the four-gene risk score distribution, OS time of patients and a heatmap showing the expression of STAC2, PRR11, HOXC11 and NUSAP1 in the training set (Fig. 3A) and the test set (Fig. 3B). In the group of patients with high-risk score, PRR11, HOXC11 and NUSAPl were upregulated, whereas $S T A C 2$ was downregulated.

Four-gene signature is an independent predictor for OS. The results of univariate Cox regression analysis for the training set and the test set demonstrated that the four-gene risk score (training set: $\mathrm{P}=3.01 \times 10^{-05}, \mathrm{HR}=1.086, \mathrm{CI}=1.044-1.128$; test set: $\mathrm{P}=0.0288, \mathrm{HR}=0.827, \mathrm{CI}=0.582-1.175)$ and the pathological stage (training set: $\mathrm{P}=0.00472, \mathrm{HR}=1.813, \mathrm{CI}=1.200-2.738$; test set: $\mathrm{P}=0.0448, \mathrm{HR}=2.646, \mathrm{CI}=1.023-3.845$ ) were significantly associated with the survival of patients with breast cancer (Table II). Furthermore, the four-gene risk score (training set: $\mathrm{P}=0.0032, \mathrm{HR}=1.069, \mathrm{CI}=1.023-1.117$; test set: $\mathrm{P}=0.0205, \mathrm{HR}=0.780, \mathrm{CI}=0.534-1.141)$ and the pathological stage (training set: $\mathrm{P}=0.0047, \mathrm{HR}=1.198, \mathrm{CI}=0.487-2.947$; test set: $\mathrm{P}=0.0663, \mathrm{HR}=2.458, \mathrm{CI}=0.941-3.42$ ) were independent predictors of survival according to the multivariate Cox regression analysis, which included clinical variables for both sets (Table II).

Correlation analysis between risk score and clinical characteristics of patients. To explore whether the prognostic power of the four-gene signature was independent of other clinical features, including age, pathologic_M, N, stage and progesterone receptor (PR) status, the data stratification analysis was carried out for each clinical feature in the training set (Table III and Figs. 4 and 5). Firstly, all hypermethylated samples of the training set were stratified by age into an older dataset ( $>60$ years, $n=132$ ) and a younger dataset $(\leq 60$ years, $n=132)$. In addition, the older dataset was classified into a high-risk group and a low-risk group: However, no significant difference in OS time was observed between the two risk groups $(\mathrm{P}=0.273$; Table III; Fig. 4A and C). The younger dataset was classified according to the risk score and divided into a high-risk and a 

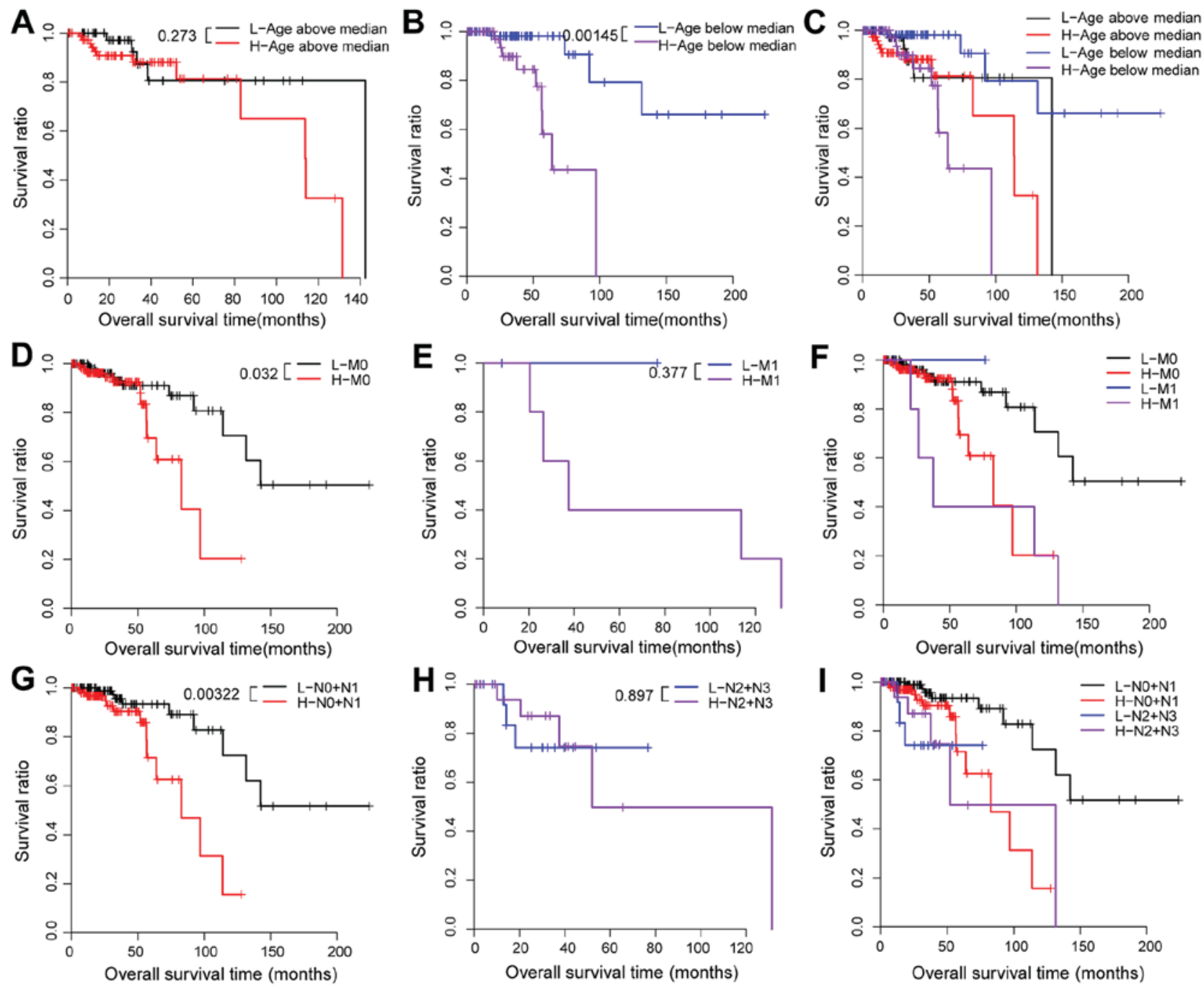

Figure 4. Kaplan-Meier plots of patients with breast cancer and hypermethylated interleukin 15 receptor $\alpha$ in The Cancer Genome Atlas set stratified by clinical features and risk score. (A-C) Patients were stratified by age into (A) an older dataset ( $>60$ years, $n=132)$ and a (B) younger dataset $(\leq 60$ years, $n=132)$, both of which were then classified by risk score into two risk groups. (C) is the merged image of (A) and (B) . (D-F) Patients were stratified by pathologic_M into an (D) M0 dataset (n=284 and an (E) M1 dataset (n=9), which were further classified into two risk groups by risk score. (F) is the merged image of (D) and (E) . (G-I) Patients were stratified by pathologic_N into an $(\mathrm{G}) \mathrm{N} 0+\mathrm{N} 1$ dataset $(\mathrm{n}=227)$ and an $(\mathrm{H}) \mathrm{N} 2+\mathrm{N} 3$ dataset $(\mathrm{n}=37)$, that were further dichotomized into two risk groups by risk score. (I) is a merged image of $(\mathrm{G})$ and $(\mathrm{H})$. P-values were calculated using the log-rank test.

Table III. Results of data stratification analysis for the training set.

\begin{tabular}{lr} 
Variable & P-value \\
\hline Age $(>60$ years, $\mathrm{n}=132)$ & 0.273 \\
Age $(<60$ years, $\mathrm{n}=132)$ & $\mathbf{< \mathbf { 0 0 5 }}$ \\
pathologic_M $(\mathrm{M} 0, \mathrm{n}=284)$ & $\mathbf{0 . 0 3 2}$ \\
pathologic_M $(\mathrm{M} 1, \mathrm{n}=9)$ & 0.377 \\
pathologic_N $(\mathrm{N} 0+\mathrm{N} 1, \mathrm{n}=227)$ & $\mathbf{0 . 0 0 5}$ \\
pathologic_N $(\mathrm{N} 2+\mathrm{N} 3, \mathrm{n}=37)$ & 0.897 \\
pathologic_stage $(\mathrm{I}+\mathrm{II}, \mathrm{n}=215)$ & $<\mathbf{0 . 0 0 5}$ \\
pathologic_stage $(\mathrm{III}+\mathrm{IV}, \mathrm{n}=49)$ & 0.612 \\
PR_Status (Positive, $\mathrm{n}=173)$ & $<\mathbf{0 . 0 0 5}$ \\
PR_Status (Negative, $\mathrm{n}=91)$ & 0.051
\end{tabular}

Bold indicates significant differences. PR, progesterone receptor. low-risk group with significantly different $\mathrm{OS}$ time $(\mathrm{P}=0.0015$; Table III, Fig. 4B and C). Subsequently, all hypermethylated samples were stratified by pathologic_M into an M0 dataset $(\mathrm{n}=284)$ and an M1 dataset $(\mathrm{n}=9)$. The M0 dataset was divided according to the risk score into a high-risk and a low-risk group. Difference in OS time between the two risk groups was significant ( $\mathrm{P}=0.032$; Table III, Fig. 4D and F). Nevertheless, the risk score that classified the M1 dataset into a high-risk and a low-risk group did not present significant difference in the OS time ( $\mathrm{P}=0.3766$; Table III, Fig. $4 \mathrm{E}$ and $\mathrm{F})$. In addition, all hypermethylated samples were stratified by pathologic_N into an $\mathrm{N0}+\mathrm{N} 1$ dataset $(\mathrm{n}=227)$ and an $\mathrm{N} 2+\mathrm{N} 3$ dataset $(\mathrm{n}=37)$. A significant difference in OS time between the high-risk and the low-risk group was observed in the $\mathrm{N} 0+\mathrm{N} 1$ dataset $(\mathrm{P}=0.0032$; Table III, Fig. 4G and I) but not in the $\mathrm{N} 2+\mathrm{N} 3$ dataset ( $\mathrm{P}=0.897$; Table III, Fig. $4 \mathrm{H}$ and I). Based on the pathologic_stage, these samples were categorized into a stage I + II dataset $(n=215)$ and a stage III + IV dataset $(n=49)$. In the stage I + II dataset, the difference in OS time was significantly 

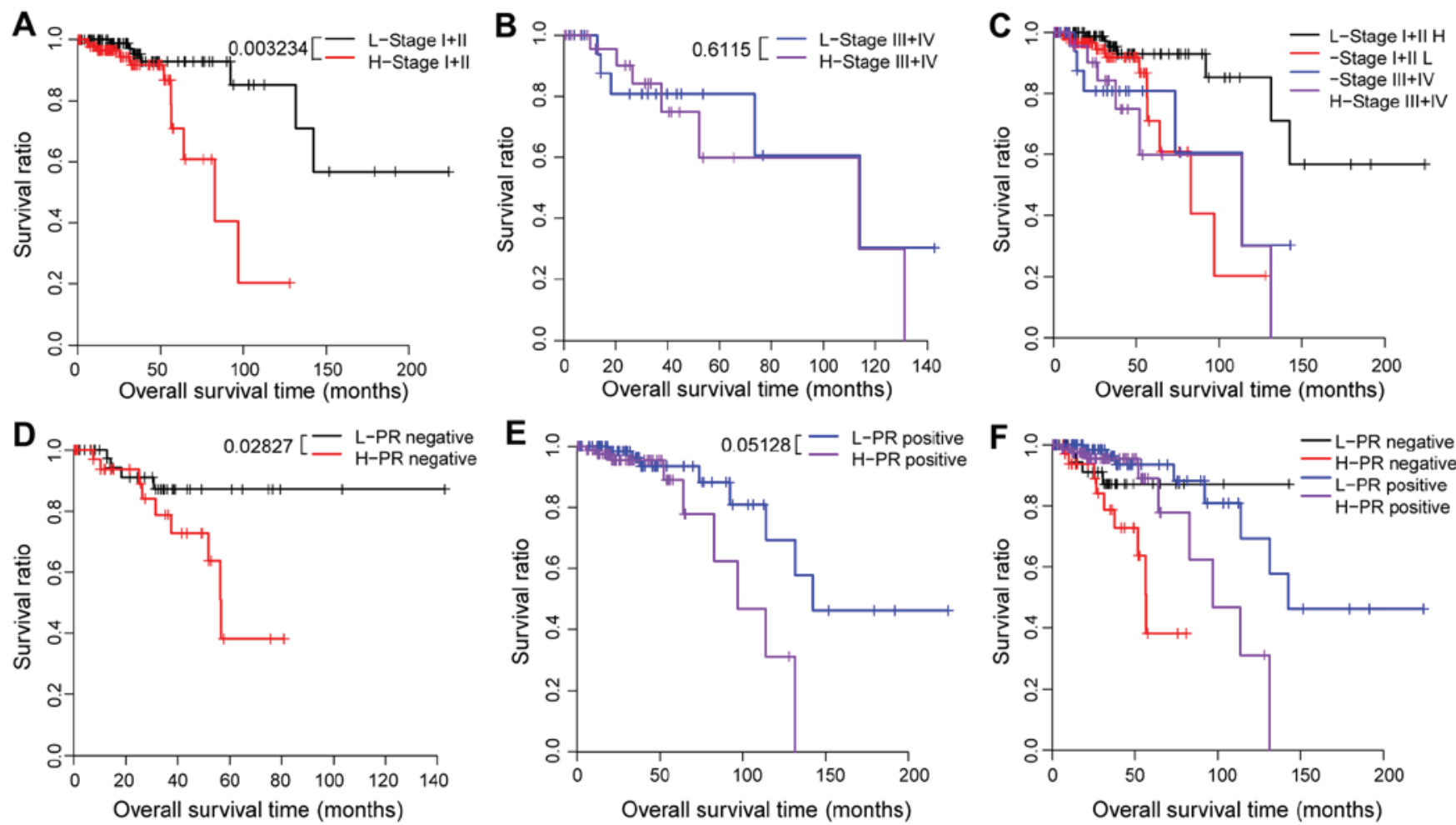

Figure 5. Kaplan-Meier plots of interleukin 15 receptor $\alpha$ hypermethylated breast cancer patients in The Cancer Genome Atlas set stratified by pathologic_stage, PR_status and risk score. (A-C) Kaplan-Meier plots of the patients at (A) stage I + II and (B) stage III + IV. (C) is the merged image of (A) and (B) . (D-F) Kaplan-Meier plots of (D) PR-negative patients (D) and (E) PR-positive patients. (F) is the merged image of (D) and (E) . P-values were calculated using the log-rank test. PR, progesterone receptor.

A

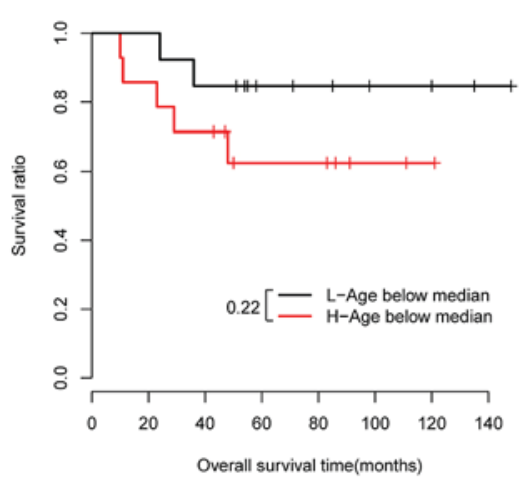

D

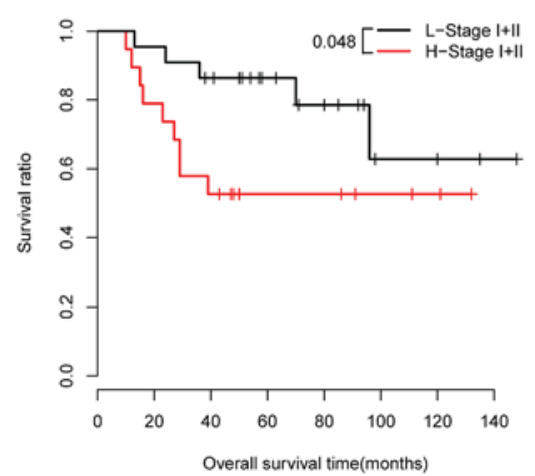

B

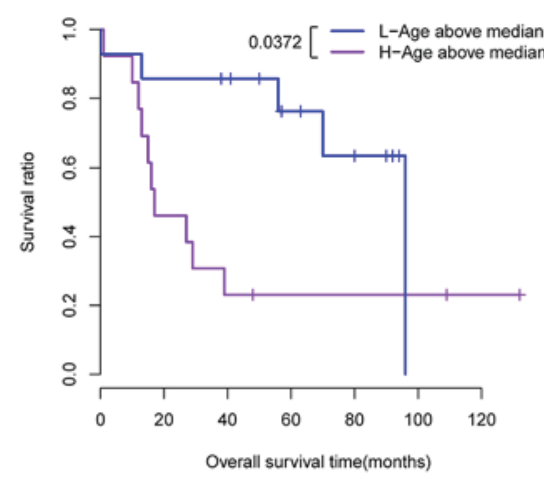

E

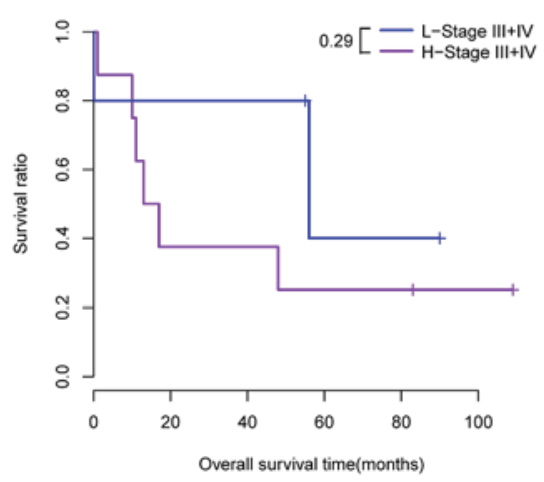

C

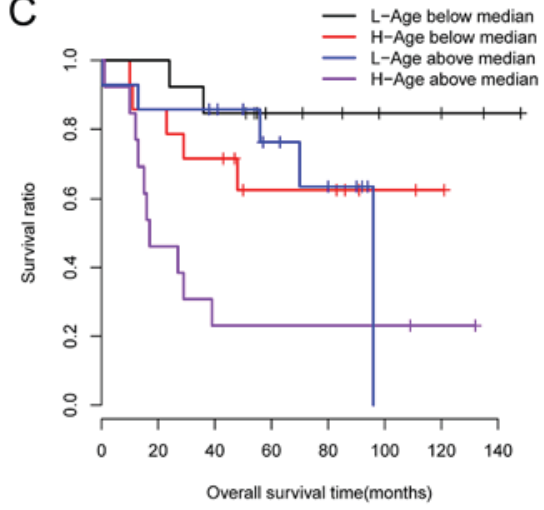

$\mathrm{F}$

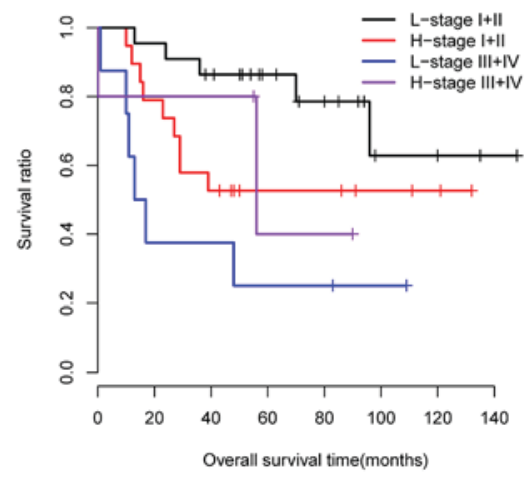

Figure 6. Kaplan-Meier plots of patients in the Gene Expression Omnibus dataset stratified by age, pathological stage and risk score. (A-C) Kaplan-Meier plots of (A) younger patients and (B) older patients. (C) is the merged image of (A) and (B) . (D-F) Kaplan-Meier plots of patients at (D) stage I + II and (E) stage III + IV. (F) is the merged image of (D) and (F) . P-values were calculated using the log-rank test. 
different between the two risk groups $(\mathrm{P}=0.0032$; Table III, Fig. 5A). However, in the stage III + IV dataset, the survival time was not significantly different between the high-risk and the low-risk group ( $\mathrm{P}=0.6115$; Table III, Fig. 5B and $\mathrm{C}$ ). These samples were eventually grouped into a PR_positive $(n=173)$ and a PR_negative dataset ( $\mathrm{n}=91)$ according to their PR_status. Difference in OS time between the high-risk and the low-risk group was significant in the $\mathrm{PR}$ _positive dataset $(\mathrm{P}=0.0283$; Table III, Fig. 5D) but not in the PR_negative dataset $(\mathrm{P}=0.0513$; Table III, Fig. 5E and F). These results indicated that the predictive capacity of the four-gene risk score for prognosis may be associated with the aforementioned clinical features.

Data stratification analysis was also conducted for the GEO dataset. All breast cancer samples in the GEO dataset were stratified by age and pathological stage (Fig. 6). The difference in the OS time between the high-risk and the low-risk group was not significant in the younger dataset $(\mathrm{P}=0.22$; Fig. $6 \mathrm{~A})$, however, was significant in the older dataset $(\mathrm{P}=0.0372$; Fig. $6 \mathrm{~B}$ and $\mathrm{C}$ ). In addition, the difference in the OS time between the high-risk and the low-risk group was significant in the stage I + II dataset $(\mathrm{P}=0.048$; Fig. 6D), however, was not significant in the stage III + IV dataset $(\mathrm{P}=0.29$; Fig. $6 \mathrm{E}$ and $\mathrm{F})$.

Functional analysis. In order to identify the molecular mechanisms underlying the prognostic performance of this four-gene signature, the present study investigated the DEGs between the high-risk and the low-risk group of the IL15RA hypermethylated samples in the training set. Subsequently, among the 308 DEGs retrieved, 224 were negatively associated with the risk score (negative DEGs) and 84 were positively associated with the risk score (positive DEGs). These positive or negative DEGs were ranked according to the correlation coefficient. A list of the top 20 positive DEGs and top 20 negative DEGs is shown in Table IV, and includes STAC2, PRRI1 and HOXC11. Hierarchical cluster analysis revealed that the gene expression pattern of high-risk samples was different from that of low-risk samples (Fig. 7A). In order to determine the possible biological roles of the top 20 positive and top 20 negative DEGs, GO function and KEGG pathway enrichment analysis were carried out. As shown in Fig. 7B, positive DEGs were mainly associated with several transcription-related GO BP terms, whereas negative DEGs were mostly associated with many cellular homeostasis-related GO $\mathrm{BP}$ terms. These genes were significantly associated with several KEGG pathways, such as 'ECM-receptor interaction' pathway and 'cell adhesion molecules (CAMs)' pathway (Fig. 7C).

\section{Discussion}

Breast cancer is a common type of cancer in woman (16). It has been reported that high expression of IL15RA contributes to better survival outcomes for patients with breast cancer (9). The present study explored the molecular mechanisms underlying the positive effect of IL15RA upregulation on patient prognosis. The results detected 326 DEGs in the hypomethylation and hypermethylation samples compared to normal samples. A four-gene signature was then identified and risk score for prognosis was calculated. The gene signature consisted of STAC2, PRR11, HOXC11 and NUSAPI. The four-gene signature-based risk score enabled classification of patients with IL15RA hypermethylated breast cancer in the training set into a high-risk and a low-risk
Table IV. Top 20 negative DEGs and top 20 positive DEGs.

\begin{tabular}{lc}
\hline A, Negative DEGs & \\
\hline Gene & Correlation coefficients \\
\hline STAC2 & -0.626 \\
SFRP1 & -0.470 \\
MSRA & -0.435 \\
KCNMB1 & -0.425 \\
SAA1 & -0.421 \\
KRT17 & -0.419 \\
PDLIM4 & -0.409 \\
TRIM29 & -0.409 \\
KRT14 & -0.409 \\
TMEM220 & -0.408 \\
SPRY2 & -0.406 \\
KRT5 & -0.400 \\
TP63 & -0.398 \\
SAA2 & -0.394 \\
COL17A1 & -0.391 \\
KLK5 & -0.389 \\
KRT15 & -0.389 \\
TINAGL1 & -0.384 \\
NTRK2 & -0.382 \\
& -0.381 \\
&
\end{tabular}

\section{B, Positive DEGs}

Gene

Correlation coefficients

\begin{tabular}{ll}
\hline PRR11 & 0.562 \\
INTS2 & 0.523 \\
SERINC5 & 0.417 \\
HOXC11 & 0.406 \\
TAOK1 & 0.401 \\
UHMK1 & 0.383 \\
BRIP1 & 0.374 \\
GAS2L3 & 0.370 \\
CASC5 & 0.358 \\
CCNT1 & 0.358 \\
DDI2 & 0.353 \\
CLSPN & 0.345 \\
HOXC13 & 0.342 \\
CENPI & 0.338 \\
HOXC10 & 0.333 \\
SHCBP1 & 0.331 \\
EPN3 & 0.329 \\
CCNE2 & 0.323 \\
ZBTB37 & 0.322 \\
E2F8 & 0.319 \\
\hline &
\end{tabular}

DEGs, differentially expressed genes. 

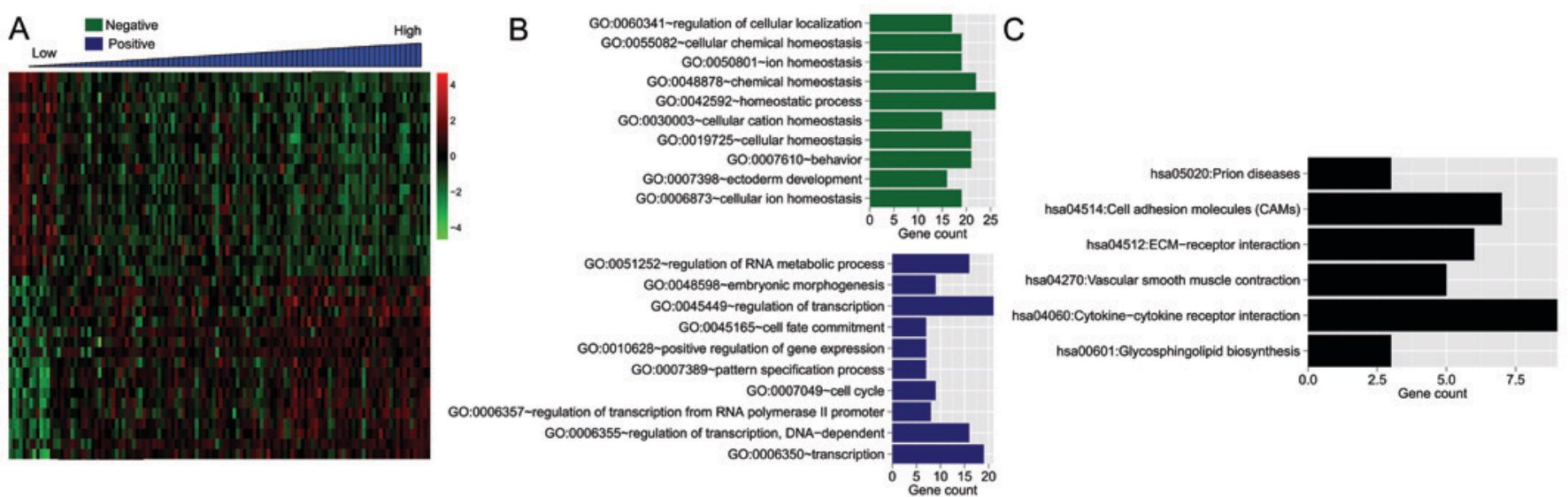

Figure 7. Results of GO function and KEGG enrichment analyses. (A) Heatmap for the top 20 positive DEGs and top 20 negative DEGs. (B) Significant GO BP terms associated with positive or negative DEGs. (C) Significant KEGG pathways enriched with both positive and negative DEGs. Gene count reflected the number of genes significantly enriched in each GO term or KEGG pathway. GO, Gene Ontology; KEGG, Kyoto Encyclopedia of Genes and Genomes.

group that had significantly different survival times. The prognostic performance of this four-gene signature-based risk score was verified in the GEO set. Furthermore, the four-gene risk score was a significant prognostic factor in the multivariate Cox regression analysis. These results suggested that the use of this four-gene signature may allow for prognostic prediction in patients with breast cancer. This also suggested that IL15RA may affect the development and progression of breast cancer partly via modulating the expression of these DEGs, thus influencing the prognosis of patients.

In the present study, the prognostic gene signature for breast cancer included STAC2, PRR11, HOXC11 and NUSAP1. STAC2 belongs to a small family of SH3 and cysteine-rich containing adaptor proteins (STAC1, STAC2 and STAC3), and is expressed in various types of tissue (17). Few reports concern STAC2 in breast cancer. PRR11 belongs to the family of proline-rich proteins. Zhou et a (18) reported that PRR11 promotes the invasion of breast cancer cells by regulating epithelial-to-mesenchymal transition (EMT). The findings from the present study suggested that IL15RA may regulate EMT by controlling PRRI1 expression. HOXCl1 is a member of the homeobox family of genes, which encode transcription factors involved in morphogenesis (19). It has been demonstrated that HOX genes serve important roles in cancer development by regulating several biological processes, including apoptosis, differentiation and angiogenesis (20). Makiyama et al (21) reported that $\mathrm{HOXC11}$ expression is decreased in breast cancer tissues compared to non-cancerous tissues. Soon et al (22) revealed that the interaction of $\mathrm{HOXC11}$ with the steroid receptor coactivator SRC-1 is responsible for the development of endocrine therapy resistance in breast cancer and suggested that nuclear HOXC11 is associated with survival. Nucleolar-spindle associated protein (NuSAP1), encoded by the NUSAPl gene, is a microtubule- and chromatin-binding protein that is critical for the spindle assembly in the cytokinesis stages of mitosis (23). Subsequently, the dysregulated expression of NuSAP1 leads to cancer development (24). A growing body of evidence also reported that NuSAP1 is associated with various types of cancer, including cervical cancer, lung adenocarcinoma and prostate cancer (25-27). Furthermore, it has been demonstrated that elevated expression of NuSAP1 is predictive of poor prognosis in triple-negative breast cancer (28). The present study also demonstrated that NuSAP1 was associated with survival of patients with breast cancer.

In the present study, the DEGs between the high-risk and the low-risk group of the training set were also investigated in order to determine the underlying mechanisms of the four-gene signature to predict prognosis. Among these DEGs, the top 20 DEGs positively or negatively associated with risk score were further selected. Results of GO analysis revealed that the top 20 positive DEGs were mainly involved in transcription-related biological processes, whereas the top 20 negative DEGs were mostly associated with cellular homeostasis-related biological processes. These DEGs were significantly associated with a number of signaling pathways, including 'ECM-receptor interaction' and 'cell adhesion molecule (CAMs)' pathways. It has been demonstrated that extracellular matrix (ECM) is involved in breast cancer development and progression (29). In addition, Emery and Tripathi reported that dysregulation of the adhesion and ECM pathways serves a role in breast cancer progression (30). A recent study by $\mathrm{He}$ et al (31) identified the role of ECM-receptor interaction in the crosstalk between tumor stroma and the peripheral blood mononuclear cells in breast cancer by using an integrated bioinformatics approach. The findings from the present study indicated that IL15RA may exert an effect on breast cancer development and progression partly by modulating 'ECM-receptor interaction' and 'cell adhesion molecules (CAMs)' pathways.

In conclusion, the present study suggested that IL15RA may affect breast cancer development and progression by regulating expression of STAC2, PRR11, HOXC11 and NUSAP1, and 'ECM-receptor interaction' and 'cell adhesion molecules (CAMs)' pathways. A four-gene signature was proposed for the prediction of prognosis in patients with breast cancer. The present study improved the understanding of the potent biological roles of IL15RA in the development of breast cancer. Further validation of this prognostic four-gene signature in a large cohort of patients with breast cancer, and experimental validation of these microarray-based results are required.

\section{Acknowledgements}

Not applicable. 


\section{Funding}

The present study was supported by Suzhou Commission of Health and Family Planning (grant no. LCZX201713) and Foundation of Nanjing Medical University (no. 2017NJMU164).

\section{Availability of data and materials}

The datasets used and/or analyzed during the present study are available from the corresponding author on reasonable request.

\section{Authors' contributions}

HY and LZ performed data analyses and wrote the manuscript. JHC, JS, WS, BL and JDZ contributed significantly to data analyses and manuscript revision. SYY and JQ conceived and designed the study. All authors read and approved the final manuscript.

\section{Ethics approval and consent to participate}

Not applicable.

\section{Patient consent for publication}

Not applicable.

\section{Competing interests}

The authors declare that they have no competing interests.

\section{References}

1. Mcguire S: World Cancer Report 2014. Geneva, Switzerland: World Health Organization, International Agency for Research on Cancer, WHO Press, 2015. Adv Nutr 7: 418-419, 2016.

2. Perou CM, Sørlie T, Eisen MB, van de Rijn M, Jeffrey SS, Rees CA, Pollack JR, Ross DT, Johnsen H, Akslen LA, et al: Molecular portraits of human breast tumours. Nature 406: 747-752, 2000.

3. Denkert C, Loibl S, Noske A, Roller M, Müller BM, Komor M, Budczies J, Darb-Esfahani S, Kronenwett R, Hanusch C, et al: Tumor-associated lymphocytes as an independent predictor of response to neoadjuvant chemotherapy in breast cancer. J Clin Oncol 28: 105-113, 2010

4. Adams S, Gray RJ, Demaria S, Goldstein L, Perez EA, Shulman LN, Martino S, Wang M, Jones VE, Saphner TJ, et al: Prognostic value of tumor-infiltrating lymphocytes in triple-negative breast cancers from two phase III randomized adjuvant breast cancer trials: ECOG 2197 and ECOG 1199. J Clin Onc 32: 2959-2966, 2014.

5. Dieci MV, Mathieu MC, Guarneri V, Conte P, Delaloge S, Andre F and Goubar A: Prognostic and predictive value of tumor-infiltrating lymphocytes in two phase III randomized adjuvant breast cancer trials. Ann Oncol 26: 1698-1704, 2015.

6. Mahmoud SM, Paish EC, Powe DG, Macmillan RD, Grainge MJ, Lee AH, Ellis IO and Green AR: Tumor-infiltrating CD8+ lymphocytes predict clinical outcome in breast cancer. J Clin Oncol 29: 1949-1955, 2011.

7. Waldmann T: The contrasting roles of IL-2 and IL-15 in the life and death of lymphocytes: Implications for the immunotherapy of rheumatological diseases. Arthritis Res 4: S161-S167, 2002.

8. Lodolce JP, Burkett PR, Koka RM, Boone DL and Ma A: Regulation of lymphoid homeostasis by interleukin-15. Cytokine Growth Factor Rev 13: 429-439, 2002.

9. Marra P, Mathew S, Grigoriadis A, Wu Y, Kyle-Cezar F, Watkins J, Rashid M, De Rinaldis E, Hessey S, Gazinska P, et al: IL15RA drives antagonistic mechanisms of cancer development and immune control in lymphocyte-enriched triple-negative breast cancers. Cancer Res 74: 4908-4921, 2014.
10. Terunuma A, Putluri N, Mishra P, Mathé EA, Dorsey TH, Yi M, Wallace TA, Issaq HJ, Zhou M, Killian JK, et al: MYC-driven accumulation of 2-hydroxyglutarate is associated with breast cancer prognosis. J Clin Invest 124: 398-412, 2014.

11. Ritchie ME, Phipson B, Wu D, Hu Y,Law CW, Shi W and Smyth GK: limma powers differential expression analyses for RNA-sequencing and microarray studies. Nucleic Acids Res 43: e47, 2015.

12. Wang P, Wang Y, Hang B, Zou X and Mao JH: A novel gene expression-based prognostic scoring system to predict survival in gastric cancer. Oncotarget 7: 55343-55351, 2016.

13. Thomas PD: The gene ontology and the meaning of biological function. Methods Mol Biol 1446: 15-24, 2017.

14. Kanehisa M, Sato Y, Kawashima M, Furumichi M and Tanabe M: KEGG as a reference resource for gene and protein annotation. Nucleic Acids Res 44: D457-D462, 2016.

15. Dennis G Jr, Sherman BT, Hosack DA, Yang J, Gao W, Lane HC and Lempicki RA: DAVID: Database for Annotation, visualization, and integrated discovery. Genome Biol 4: P3, 2003.

16. Desantis C, Ma J, Bryan L and Jemal A: Breast cancer statistics, 2013. CA Cancer J Clin 64: 52-62, 2014.

17. Nelson BR, Wu F, Liu Y, Anderson DM, McAnally J, Lin W, Cannon SC, Bassel-Duby R and Olson EN: Skeletal muscle-specific T-tubule protein STAC3 mediates voltage-induced $\mathrm{Ca} 2+$ release and contractility. Proc Natl Acad Sci USA 110: 11881-11886, 2013.

18. Zhou F, Liu H, Zhang X, Shen Y, Zheng D, Zhang A, Lai Y and Li H: Proline-rich protein 11 regulates epithelial-to-mesenchymal transition to promote breast cancer cell invasion. Int J Clin Exp Pathol 7: 8692-8699, 2014.

19. Holland PWH: Evolution of homeobox genes. Wiley Interdisciplinary Rev Dev Biol 2: 31-45, 2013.

20. Bhatlekar S, Fields JZ and Boman BM: HOX genes and their role in the development of human cancers. J Mol Med (Berl) 92: 811-823, 2014.

21. Makiyama K, Hamada J, Takada M, Murakawa K, Takahashi Y, Tada M, Tamoto E, Shindo G, Matsunaga A, Teramoto K, et al: Aberrant expression of HOX genes in human invasive breast carcinoma. Oncol Rep 13: 673-679, 2005.

22. Soon YH, Bane F, Hughes E and Young LS: Protein bomarkers $\mathrm{Ki67}, \mathrm{HOXC10}$ and $\mathrm{HOXC11}$ for the prediction of response to endocrine treatment in breast cancer. BMC Proc 9: A52, 2015.

23. Ribbeck K, Groen AC, Santarella R, Bohnsack MT, Raemaekers T, Köcher T, Gentzel M, Görlich D, Wilm M, Carmeliet G, et al: NuSAP, a Mitotic RanGTP target that stabilizes and cross-links microtubules. Mol Biol Cell 17: 2646-2660, 2006.

24. Iyer J, Moghe S, Furukawa M and Tsai MY: What's Nu(SAP) in mitosis and cancer? Cell Signal 23: 991-998, 2011.

25. Bidkhori G, NarimaniZ, Ashtiani SH, Moeini A, Nowzari-Dalini A and Masoudi-Nejad A: Reconstruction of an integrated genome-scale co-expression network reveals key modules involved in lung adenocarcinoma. PLoS One 8: e67552, 2013.

26. Espinosa AM, Alfaro A, Roman-Basaure E, Guardado-Estrada M, Palma Í,Serralde C,Medina I,JuárezE,Bermúdez M,MárquezE, et al: Mitosis is a source of potential markers for screening and survival and therapeutic targets in cervical cancer. PLoS One 8: e55975, 2013.

27. Gulzar ZG, Mckenney JK and Brooks JD: Increased expression of NuSAP in recurrent prostate cancer is mediated by E2F1. Oncogene 32: 70-77, 2013.

28. Chen L, Yang L, Qiao F, Hu X, Li S, Yao L, Yang XL and Shao ZM: High levels of nucleolar spindle-associated protein and reduced levels of BRCA1 expression predict poor prognosis in triple-negative breast cancer. PLoS One 10: e0140572, 2015.

29. Giussani M, Merlino G, Cappelletti V, Tagliabue E and Daidone MG: Tumor-extracellular matrix interactions: Identification of tools associated with breast cancer progression. Semin Cancer Biol 35: 3-10, 2015.

30. Emery LA, Tripathi AC, King C, Kavanah M, Mendez J, Stone MD, de las Morenas A, Sebastiani P and Rosenberg CL: Early dysregulation of cell adhesion and extracellular matrix pathways in breast cancer progression. Am J Pathol 175: 1292-1302, 2009.

31. He L, Wang D, Wei N and Guo Z: Integrated bioinformatics approach reveals crosstalk between tumor stroma and peripheral blood mononuclear cells in breast cancer. Asian Pac J Cancer Prev 17: 1003-1008, 2016.

(i) $($ This work is licensed under a Creative Commons Attribution-NonCommercial-NoDerivatives 4.0 International (CC BY-NC-ND 4.0) License. 